\title{
On Some Symmetric Systems of Difference Equations
}

\author{
Josef Diblík, ${ }^{1,2}$ Bratislav Iričanin, ${ }^{3}$ Stevo Stevic,, ${ }^{4,5}$ and Zdeněk Šmarda ${ }^{2}$ \\ ${ }^{1}$ Department of Mathematics and Descriptive Geometry, Faculty of Civil Engineering, Brno University of Technology, \\ 60200 Brno, Czech Republic \\ ${ }^{2}$ Department of Mathematics, Faculty of Electrical Engineering and Communication, Brno University of Technology, \\ 61600 Brno, Czech Republic \\ ${ }^{3}$ Faculty of Electrical Engineering, University of Belgrade, Bulevar Kralja Aleksandra 73, 11000 Beograd, Serbia \\ ${ }^{4}$ Mathematical Institute of the Serbian Academy of Sciences, Knez Mihailova 36/III, 11000 Beograd, Serbia \\ ${ }^{5}$ Department of Mathematics, King Abdulaziz University, Jeddah 21859, Saudi Arabia
}

Correspondence should be addressed to Stevo Stević; sstevic@ptt.rs

Received 22 December 2012; Accepted 12 February 2013

Academic Editor: Norio Yoshida

Copyright (C) 2013 Josef Diblík et al. This is an open access article distributed under the Creative Commons Attribution License, which permits unrestricted use, distribution, and reproduction in any medium, provided the original work is properly cited.

Here we show that the main results in the papers by Yalcinkaya (2008), Yalcinkaya and Cinar (2010), and Yalcinkaya, Cinar, and Simsek (2008), as well as a conjecture from the last mentioned paper, follow from a slight modification of a result by G. Papaschinopoulos and C. J. Schinas. We also give some generalizations of these results.

\section{Introduction}

Studying difference equations and systems which possess some kind of symmetry attracted some attention recently (see, e.g., [1-25] and the related references therein).

Paper [23] studied the following system of difference equations:

$$
\begin{gathered}
x_{n+1}=\frac{y_{n} x_{n-1}+a}{y_{n}+x_{n-1}}, \\
y_{n+1}=\frac{x_{n} y_{n-1}+a}{x_{n}+y_{n-1}}, \quad n \in \mathbb{N}_{0} .
\end{gathered}
$$

In [24], authors claim that they study the system

$$
\begin{gathered}
x_{n+1}=\frac{y_{n}+x_{n-1}}{y_{n} x_{n-1}+a}, \\
y_{n+1}=\frac{x_{n}+y_{n-1}}{x_{n} y_{n-1}+a}, \quad n \in \mathbb{N}_{0},
\end{gathered}
$$

while in [25], the authors studied the system

$$
\begin{gathered}
x_{n+1}=\frac{x_{n} y_{n-1}+a}{x_{n}+y_{n-1}}, \\
y_{n+1}=\frac{y_{n} x_{n-1}+a}{y_{n}+x_{n-1}}, \quad n \in \mathbb{N}_{0},
\end{gathered}
$$

where $a>0$.

Since $a>0$, it is clear that the change of variables

$$
\left(x_{n}, y_{n}\right) \longrightarrow\left(\sqrt{a} x_{n} \sqrt{a} y_{n}\right),
$$

reduces systems (1) and (3) to the case $a=1$. The authors of [24] claim that the change of variables (4) reduces (2) to the case $a=1$ too; however by using the change system (2) becomes

$$
\begin{gathered}
x_{n+1}=\frac{y_{n}+x_{n-1}}{a\left(y_{n} x_{n-1}+1\right)}, \\
y_{n+1}=\frac{x_{n}+y_{n-1}}{a\left(x_{n} y_{n-1}+1\right)}, \quad n \in \mathbb{N}_{0} .
\end{gathered}
$$

Therefore, in fact, [24] studied only system (2) for the case $a=1$. 
Based on this observation we may, and will, assume that $a=1$ in systems of difference equations (1)-(3).

In the main results in [23-25] it is proved that when $a=1$, the positive equilibrium point $(\bar{x}, \bar{y})=(1,1)$ of systems $(1)-$ (3) is globally asymptotically stable.

The authors of [25] finish their paper by the statement that they believe that the results therein can be conveniently extended to the following higher order system of difference equations:

$$
\begin{gathered}
x_{n+1}=\frac{x_{n} y_{n-l}+a}{x_{n}+y_{n-l}}, \\
y_{n+1}=\frac{y_{n} x_{n-l}+a}{y_{n}+x_{n-l}}, \quad n \in \mathbb{N}_{0},
\end{gathered}
$$

when $l \in \mathbb{N} \backslash\{1\}$.

Here, among others, we show that all the results and conjectures mentioned above follow from a slight modification of a result in the literature published before papers [23-25]. For related systems see also [2, 5-10, 12, 17-20].

\section{Main Results}

Let $\mathbb{R}_{+}=(0,+\infty)$ and $\mathbb{R}_{+}^{n}$ be the set of all positive $n$ dimensional vectors. The following theorem was proved in [4].

Theorem A. Let $(M, d)$ be a complete metric space, where $d$ denotes a metric and $M$ is an open subset of $\mathbb{R}^{n}$, and let $T: M \rightarrow M$ be a continuous mapping with the unique equilibrium $x^{*} \in M$. Suppose that for the discrete dynamic system

$$
x_{n+1}=T x_{n}, \quad n \in \mathbb{N}_{0},
$$

there is a $k \in \mathbb{N}$ such that for the $k$ th iterate of $T$, the following inequality holds:

$$
d\left(T^{k} x, x^{*}\right)<d\left(x, x^{*}\right)
$$

for all $x \neq x^{*}$. Then $x^{*}$ is globally asymptotically stable with respect to metric $d$.

The part-metric (see [21]) is a metric defined on $\mathbb{R}_{+}^{n}$ by

$$
p(X, Y)=-\log \min _{1 \leq i \leq n}\left\{\frac{x_{i}}{y_{i}}, \frac{y_{i}}{x_{i}}\right\}
$$

for arbitrary vectors $X=\left(x_{1}, x_{2}, \ldots, x_{n}\right)^{T} \in \mathbb{R}_{+}^{n}$ and $Y=$ $\left(y_{1}, y_{2}, \ldots, y_{n}\right)^{T} \in \mathbb{R}_{+}^{n}$.

It is known that the part-metric $p$ is a continuous metric on $\mathbb{R}_{+}^{n},\left(\mathbb{R}_{+}^{n}, p\right)$ is a complete metric space, and that the distances induced by the part-metric and by the Euclidean norm are equivalent on $\mathbb{R}_{+}^{n}$ (see, e.g., [4]).

Based on these properties and Theorem A, the following corollary follows.

Corollary 1. Let $T: \mathbb{R}_{+}^{n} \rightarrow \mathbb{R}_{+}^{n}$ be a continuous mapping with a unique equilibrium $x^{*} \in \mathbb{R}_{+}^{n}$. Suppose that for the discrete dynamic system (7), there is some $k \in \mathbb{N}$ such that for the partmetric $p$ inequality

$$
p\left(T^{k} x, x^{*}\right)<p\left(x, x^{*}\right)
$$

holds for all $x \neq x^{*}$. Then $x^{*}$ is globally asymptotically stable.

Some applications of various part-metric-related inequalities and some asymptotic methods in studying difference equations related to symmetric ones can be found, for example, in $[1,3-5,10,11,13-16,22]$ (see also the related references therein).

In Lemma 2.3 in [10], Papaschinopoulos and Schinas formulated a variant of the following result, without giving a proof. However, the part concerning the equality in inequality (12) below, is not mentioned, but it is crucial in applying Corollary 1 (see inequality (10)). For this reason, the completeness and the benefit of the reader we will give a complete proof of it.

Proposition 2. Let $f: \mathbb{R}_{+}^{2 m} \rightarrow \mathbb{R}_{+}, g: \mathbb{R}_{+}^{2 m} \rightarrow \mathbb{R}_{+}$ be continuous functions. We suppose that the system of two difference equations,

$$
\begin{aligned}
& u_{n+m}=f\left(u_{n}, u_{n+1}, \ldots, u_{n+m-1}, v_{n}, v_{n+1}, \ldots, v_{n+m-1}\right), \\
& v_{n+m}=g\left(u_{n}, u_{n+1}, \ldots, u_{n+m-1}, v_{n}, v_{n+1}, \ldots, v_{n+m-1}\right),
\end{aligned}
$$

has a unique positive equilibrium $(w, w)$. Suppose also that there is an $r \in \mathbb{N}$ such that for any positive solution $\left(u_{n}, v_{n}\right)_{n \in \mathbb{N}_{0}}$ of system (11), the following inequalities:

$$
\begin{gathered}
\left(u_{n}-u_{n+r}\right)\left(\frac{w^{2}}{u_{n}}-u_{n+r}\right) \leq 0, \\
\left(v_{n}-v_{n+r}\right)\left(\frac{w^{2}}{v_{n}}-v_{n+r}\right) \leq 0, \quad n \in \mathbb{N}_{0},
\end{gathered}
$$

hold, with the equalities if and only if $u_{n}=w$, for every $n \in \mathbb{N}_{0}$, and $v_{n}=w$, for every $n \in \mathbb{N}_{0}$, respectively. Then the equilibrium $(w, w)$ is globally asymptotically stable.

Proof. First, we prove that for every $n \in \mathbb{N}_{0}$

$$
\min \left\{\frac{u_{n+r}}{w}, \frac{v_{n+r}}{w}, \frac{w}{u_{n+r}}, \frac{w}{v_{n+r}}\right\}>\min \left\{\frac{u_{n}}{w}, \frac{v_{n}}{w}, \frac{w}{u_{n}}, \frac{w}{v_{n}}\right\},
$$

if and only if $\left(u_{n}, v_{n}\right) \neq(w, w)$.

To prove (13), it is enough to prove that

$$
\min \left\{\frac{u_{n+r}}{w}, \frac{w}{u_{n+r}}\right\}>\min \left\{\frac{u_{n}}{w}, \frac{w}{u_{n}}\right\},
$$

if and only if $u_{n} \neq w$, and

$$
\min \left\{\frac{v_{n+r}}{w}, \frac{w}{v_{n+r}}\right\}>\min \left\{\frac{v_{n}}{w}, \frac{w}{v_{n}}\right\},
$$

if and only if $v_{n} \neq w$. 
The proofs of inequalities (14) and (15) are the same (up to the interchanging letters $u$ and $v$ ) so it is enough to prove (14).

Now note that if the equality holds in the first inequality in (12), then we have that

$$
u_{n}=u_{n+r} \quad \text { or } \quad \frac{w}{u_{n}}=\frac{u_{n+r}}{w},
$$

from which, in both cases, it easily follows that

$$
\min \left\{\frac{u_{n+r}}{w}, \frac{w}{u_{n+r}}\right\}=\min \left\{\frac{u_{n}}{w}, \frac{w}{u_{n}}\right\} .
$$

On the other hand, if (17) holds, then we easily obtain that one of the equalities in (16) holds, and consequently it follows that the equality holds in the first inequality in (12). Hence, by one of the assumptions, we have that (17) holds if and only if $u_{n}=w$ for every $n \in \mathbb{N}_{0}$.

Now suppose that the first inequality in (12), is strict. Then, if $u_{n}>u_{n+r}$, directly follows that $w / u_{n+r}>w / u_{n}$, while from the first inequality in (12) it follows that $u_{n+r} / w>w / u_{n}$. Hence

$$
\min \left\{\frac{u_{n+r}}{w}, \frac{w}{u_{n+r}}\right\}>\frac{w}{u_{n}},
$$

from which inequality (14) easily follows.

If $u_{n}<u_{n+r}$, then $u_{n+r} / w>u_{n} / w$, while from the first inequality in (12), it follows that $w / u_{n+r}>u_{n} / w$. From these two inequalities, we have that

$$
\min \left\{\frac{u_{n+r}}{w}, \frac{w}{u_{n+r}}\right\}>\frac{u_{n}}{w},
$$

and consequently (14).

If (14) and (15) hold then if $u_{n} \neq w$ and $v_{n} \neq w$, inequality (13) immediately follows by using the following elementary implication: if $a>b$ and $c>d$, then $\min \{a, c\}>\min \{b, d\}$.

If $u_{n} \neq w$ and $v_{n}=w$, then from the second inequality in (12), we have that $v_{n+r}=v_{n}=w$. Hence

$$
\min \left\{\frac{v_{n+r}}{w}, \frac{w}{v_{n+r}}\right\}=\min \left\{\frac{v_{n}}{w}, \frac{w}{v_{n}}\right\}=1>\min \left\{\frac{u_{n}}{w}, \frac{w}{u_{n}}\right\},
$$

which along with (14) implies (13). The case $u_{n}=w$ and $v_{n} \neq w$ directly follows from the case $u_{n} \neq w$ and $v_{n}=w$, by the symmetry.

Finally, note that if $u_{n}=v_{n}=w$, then from (12), we have that $u_{n+r}=u_{n}=w$ and $v_{n+r}=v_{n}=w$, so that the first equality in (20) holds and

$$
\min \left\{\frac{u_{n+r}}{w}, \frac{w}{u_{n+r}}\right\}=\min \left\{\frac{u_{n}}{w}, \frac{w}{u_{n}}\right\}=1,
$$

from which it follows that both minima in (13) are equal, finishing the proof of the claim.

Now we define the map $T: \mathbb{R}_{+}^{2 m} \rightarrow \mathbb{R}_{+}^{2 m}$ as follows:

$$
\begin{array}{r}
T\left(x_{1}, x_{2}, \ldots, x_{m-1}, x_{m}, y_{1}, y_{2}, \ldots, y_{m-1}, y_{m}\right) \\
=\left(x_{2}, \ldots, x_{m}, f\left(x_{1}, \ldots, x_{m}, y_{1}, \ldots, y_{m}\right),\right. \\
\left.y_{2}, \ldots, y_{m}, g\left(x_{1}, \ldots, x_{m}, y_{1}, \ldots, y_{m}\right)\right) .
\end{array}
$$

Then we get

$$
\begin{gathered}
T\left(u_{n}, u_{n+1}, \ldots, u_{n+m-2}, u_{n+m-1}, v_{n}, v_{n+1}, \ldots, v_{n+m-2}, v_{n+m-1}\right) \\
=\left(u_{n+1}, \ldots, u_{n+m-1}, u_{n+m}, v_{n+1}, \ldots, v_{n+m-1}, v_{n+m}\right),
\end{gathered}
$$

and by induction

$$
\begin{aligned}
T^{s} & \left(u_{n}, u_{n+1}, \ldots, u_{n+m-2}, u_{n+m-1}, v_{n}, v_{n+1}, \ldots, v_{n+m-2}, v_{n+m-1}\right) \\
= & \left(u_{n+s}, \ldots, u_{n+m-2+s}, u_{n+m-1+s},\right. \\
& \left.v_{n+s}, \ldots, v_{n+m-2+s}, v_{n+m-1+s}\right),
\end{aligned}
$$

for every $s \in \mathbb{N}$.

By using inequality (13) and the fact that the inequalities $1 \geq a_{i}>b_{i}, i \in I \subseteq\{1, \ldots, m\}, I \neq \emptyset$, along with equalities $a_{i}=$ $b_{i}=1, i \in\{1, \ldots, m\} \backslash I$, imply the inequality $\min _{1 \leq i \leq m} a_{i}>$ $\min _{1 \leq i \leq m} b_{i}$, we have that for each vector $\vec{x} \in \mathbb{R}_{+}^{2 m}$ such that $\vec{x} \neq(w, w, \ldots, w)=: \vec{w} \in \mathbb{R}_{+}^{2 m}$,

$$
\begin{aligned}
& p\left(T^{r}(\vec{x}), \vec{w}\right)=-\log \min \left\{\frac{u_{n+r}}{w}, \frac{w}{u_{n+r}}, \ldots, \frac{u_{n+r+m-1}}{w},\right. \\
& \frac{w}{u_{n+r+m-1}}, \frac{v_{n+r}}{w}, \frac{w}{v_{n+r}}, \ldots, \\
&\left.\frac{v_{n+r+m-1}}{w}, \frac{w}{v_{n+r+m-1}}\right\} \\
&<-\log \min \left\{\frac{u_{n}}{w}, \frac{w}{u_{n}}, \ldots, \frac{u_{n+m-1}}{w}, \frac{w}{u_{n+m-1}},\right. \\
&\left.=p(\vec{x}, \vec{w}), \quad \frac{v_{n}}{w}, \frac{w}{v_{n}}, \ldots, \frac{v_{n+m-1}}{w}, \frac{w}{v_{n+m-1}}\right\}
\end{aligned}
$$

from which the proof follows by Corollary 1.

It is not difficult to see that the following extension of Proposition 2 can be proved by slight modifications of the proof of Proposition 2.

Proposition 3. Let $f_{i}: \mathbb{R}_{+}^{l m} \rightarrow \mathbb{R}_{+}, i=1, \ldots, l$, be continuous functions. Suppose that the system of difference equations

$$
u_{n+m}^{(1)}=f_{1}\left(u_{n}^{(1)}, u_{n+1}^{(1)}, \ldots, u_{n+m-1}^{(1)}, \ldots, u_{n}^{(l)}, u_{n+1}^{(l)}, \ldots, u_{n+m-1}^{(l)}\right),
$$

$$
\begin{gathered}
u_{n+m}^{(i)}=f_{i}\left(u_{n}^{(1)}, u_{n+1}^{(1)}, \ldots, u_{n+m-1}^{(1)}, \ldots, u_{n}^{(l)}, u_{n+1}^{(l)}, \ldots, u_{n+m-1}^{(l)}\right), \\
\vdots \\
u_{n+m}^{(l)}=f_{l}\left(u_{n}^{(1)}, u_{n+1}^{(1)}, \ldots, u_{n+m-1}^{(1)}, \ldots, u_{n}^{(l)}, u_{n+1}^{(l)}, \ldots, u_{n+m-1}^{(l)}\right)
\end{gathered}
$$


has a unique positive equilibrium $(w, \ldots, w) \in \mathbb{R}_{+}^{l}$, and that there is an $r \in \mathbb{N}$ such that for any solution $\left(u_{n}^{(1)}, \ldots, u_{n}^{(l)}\right)_{n \in \mathbb{N}_{0}} \subset$ $\mathbb{R}_{+}^{l}$ of system (26), the following inequalities:

$$
\left(u_{n}^{(i)}-u_{n+r}^{(i)}\right)\left(\frac{w^{2}}{u_{n}^{(i)}}-u_{n+r}^{(i)}\right) \leq 0, \quad n \in \mathbb{N}_{0}, i=1, \ldots, l,
$$

hold, with the equalities if and only if $u_{n}^{(i)}=w$, for every $n \in \mathbb{N}_{0}$, and $i=1, \ldots, l$. Then the equilibrium $(w, \ldots, w)$ is globally asymptotically stable.

Now we use Proposition 2 in proving the results in papers [23-25].

Corollary 4. Let $k, l \in \mathbb{N}_{0}, k \neq l$. Consider the system

$$
\begin{gathered}
x_{n+1}=\frac{x_{n-k} y_{n-l}+1}{x_{n-k}+y_{n-l}}, \\
y_{n+1}=\frac{y_{n-k} x_{n-l}+1}{y_{n-k}+x_{n-l}}, \quad n \in \mathbb{N}_{0} .
\end{gathered}
$$

Then the positive equilibrium point $(\bar{x}, \bar{y})=(1,1)$ of system (28) is globally asymptotically stable with respect to the set $\mathbb{R}_{+}^{m} \times \mathbb{R}_{+}^{m}$, where $m=\max \{k, l\}$.

Proof. We may assume that $m=k$. From system (28), we have that

$$
\begin{gathered}
x_{n+1}-x_{n-k}=\frac{1-x_{n-k}^{2}}{y_{n-l}+x_{n-k}}, \\
x_{n+1}-\frac{1}{x_{n-k}}=\frac{y_{n-l}\left(x_{n-k}^{2}-1\right)}{x_{n-k}\left(y_{n-l}+x_{n-k}\right)}, \\
y_{n+1}-y_{n-k}=\frac{1-y_{n-k}^{2}}{x_{n-l}+y_{n-k}}, \\
y_{n+1}-\frac{1}{y_{n-k}}=\frac{x_{n-l}\left(y_{n-k}^{2}-1\right)}{y_{n-k}\left(x_{n-l}+y_{n-k}\right)}
\end{gathered}
$$

from which it follows that

$$
\begin{aligned}
& \left(x_{n+1}-x_{n-k}\right)\left(x_{n+1}-\frac{1}{x_{n-k}}\right) \leq 0, \\
& \left(y_{n+1}-y_{n-k}\right)\left(y_{n+1}-\frac{1}{y_{n-k}}\right) \leq 0
\end{aligned}
$$

so that condition (12) in Proposition 2 is fulfilled with $r=$ $k+1$.

Clearly if

$$
x_{n}=1=y_{n} \quad \text { for every } n \geq-\max \{l, k\},
$$

then in (31) equalities follow. On the other hand, if equality holds in the first inequality in (31), we have that

$$
x_{n+1}=x_{n-k} \quad \text { or } \quad x_{n+1}=\frac{1}{x_{n-k}} .
$$

If $x_{n+1}=x_{n-k}$, then from the first equality in (29) we have that $x_{n-k}=1$, while if $x_{n+1}=1 / x_{n-k}$, then from the second equality in (29), we have that $x_{n-k}=1$.

By symmetry (see (30)), we have that if equality holds in the second inequality in (31), then $y_{n-k}=1$. Therefore, equalities in (31) hold if and only if $\left(x_{n-k}, y_{n-k}\right)=(1,1)$. Hence all the conditions of Proposition 2 are fulfilled from which it follows that the positive equilibrium $(1,1)$ is globally asymptotically stable with respect to the set $\mathbb{R}_{+}^{m} \times \mathbb{R}_{+}^{m}$.

Remark 5. Corollary 4 extends and gives a very short proof of the main result in [23], which is obtained for $k=1$ and $l=0$. Further, it also extends and gives a very short proof of the main result in [25], which is obtained for $k=0$ and $l=1$. Moreover, it confirms the conjecture in [25], which is obtained for $k=0$ and $l \in \mathbb{N} \backslash\{1\}$.

Corollary 6. Let $k, l \in \mathbb{N}_{0}, k \neq l$. Consider the system

$$
\begin{gathered}
x_{n+1}=\frac{x_{n-k}+y_{n-l}}{x_{n-k} y_{n-l}+1}, \\
y_{n+1}=\frac{y_{n-k}+x_{n-l}}{y_{n-k} x_{n-l}+1}, \quad n \in \mathbb{N}_{0} .
\end{gathered}
$$

Then the positive equilibrium point $(\bar{x}, \bar{y})=(1,1)$ of system (34) is globally asymptotically stable with respect to the set $\mathbb{R}_{+}^{m} \times \mathbb{R}_{+}^{m}$, where $m=\max \{k, l\}$.

Proof. We may assume that $m=k$. From system (34), we have that

$$
\begin{gathered}
x_{n+1}-x_{n-k}=\frac{y_{n-l}\left(1-x_{n-k}^{2}\right)}{x_{n-k} y_{n-l}+1}, \\
x_{n+1}-\frac{1}{x_{n-k}}=\frac{x_{n-k}^{2}-1}{x_{n-k}\left(x_{n-k} y_{n-l}+1\right)}, \\
y_{n+1}-y_{n-k}=\frac{x_{n-l}\left(1-y_{n-k}^{2}\right)}{x_{n-l} y_{n-k}+1}, \\
y_{n+1}-\frac{1}{y_{n-k}}=\frac{y_{n-k}^{2}-1}{y_{n-k}\left(x_{n-l} y_{n-k}+1\right)}
\end{gathered}
$$

from which it follows that

$$
\begin{aligned}
& \left(x_{n+1}-x_{n-k}\right)\left(x_{n+1}-\frac{1}{x_{n-k}}\right) \leq 0, \\
& \left(y_{n+1}-y_{n-k}\right)\left(y_{n+1}-\frac{1}{y_{n-k}}\right) \leq 0 .
\end{aligned}
$$

Hence condition (12) in Proposition 2 is fulfilled with $r=$ $k+1$. On the other hand, similarly as in the proof of Corollary 4 it is proved that equalities in (36) hold if and only if $\left(x_{n-k}, y_{n-k}\right)=(1,1)$. Hence all the conditions of Proposition 2 are fulfilled from which it follows that the positive equilibrium $(1,1)$ is globally asymptotically stable with respect to the set $\mathbb{R}_{+}^{m} \times \mathbb{R}_{+}^{m}$. 
Remark 7. Corollary 6 extends and gives a very short proof of the main result in [24], which is obtained for $k=1$ and $l=0$.

Remark 8. Corollary 6 is also a consequence of Corollary 4. Namely, by using the change of variables $\left(x_{n}, y_{n}\right)=$ $\left(1 / u_{n}, 1 / v_{n}\right)$, system (34) is transformed into the system

$$
\begin{gathered}
v_{n+1}=\frac{v_{n-k} u_{n-l}+1}{v_{n-k}+u_{n-l}}, \\
u_{n+1}=\frac{u_{n-k} v_{n-l}+1}{u_{n-k}+v_{n-l}}, \quad n \in \mathbb{N}_{0},
\end{gathered}
$$

which is system (28). In particular, this shows that systems (1) and (2), for the case $a=1$, are equivalent and consequently the results in $[23,24]$.

Remark 9. Similar type of issues appear in some literature on scalar difference equations (see, e.g., related results in papers $[1,5,11,13])$.

It is of some interest to extend results in Corollaries 4 and 6 by using Proposition 2. The next result is of this kind and it extends a result in [5].

Corollary 10. Let $f \in C\left(\mathbb{R}_{+}^{k}, \mathbb{R}_{+}\right)$and $g \in C\left(\mathbb{R}_{+}^{l}, \mathbb{R}_{+}\right)$with $k, l \in \mathbb{N}, 0 \leq r_{1}<\cdots<r_{k}$ and $0 \leq m_{1}<\cdots<m_{l} \leq r_{k}$ and satisfy the following two conditions:

(H1) $\left[f\left(u_{1}, u_{2}, \ldots, u_{k}\right)\right]^{*}=f\left(u_{1}^{*}, u_{2}^{*}, \ldots, u_{k}^{*}\right)$,

(H2) $f\left(u_{1}^{*}, u_{2}^{*}, \ldots, u_{k}^{*}\right) \leq u_{k}^{*}$,

where $a^{*}:=\max \{a, 1 / a\}$.

Then $(\bar{x}, \bar{y})=(1,1)$ is the unique positive equilibrium of the system of difference equations

$$
\begin{array}{r}
x_{n}=\frac{f\left(x_{n-r_{1}-1}, \ldots, x_{n-r_{k}-1}\right) g\left(y_{n-m_{1}-1}, \ldots, y_{n-m_{l}-1}\right)+1}{f\left(x_{n-r_{1}-1}, \ldots, x_{n-r_{k}-1}\right)+g\left(y_{n-m_{1}-1}, \ldots, y_{n-m_{l}-1}\right)}, \\
n \in \mathbb{N}, \\
y_{n}=\frac{f\left(y_{n-r_{1}-1}, \ldots, y_{n-r_{k}-1}\right) g\left(x_{n-m_{1}-1}, \ldots, x_{n-m_{l}-1}\right)+1}{f\left(y_{n-r_{1}-1}, \ldots, y_{n-r_{k}-1}\right)+g\left(x_{n-m_{1}-1}, \ldots, x_{n-m_{l}-1}\right)},
\end{array}
$$

and it is globally asymptotically stable.

Proof. Let

$$
\begin{aligned}
& f_{n}=f\left(x_{n-r_{1}-1}, \ldots, x_{n-r_{k}-1}\right), \\
& g_{n}=g\left(y_{n-m_{1}-1}, \ldots, y_{n-m_{l}-1}\right) .
\end{aligned}
$$

We should determine the sign of the product of the following expressions:

$$
\begin{aligned}
P_{n} & :=\frac{f_{n} g_{n}+1}{f_{n}+g_{n}}-x_{n-r_{k}-1} \\
& =\frac{1}{f_{n}+g_{n}}\left(f_{n} g_{n}\left(1-\frac{x_{n-r_{k}-1}}{f_{n}}\right)+1-x_{n-r_{k}-1} f_{n}\right), \\
Q_{n} & :=\frac{f_{n} g_{n}+1}{f_{n}+g_{n}}-\frac{1}{x_{n-r_{k}-1}} \\
= & \frac{1}{x_{n-r_{k}-1}\left(f_{n}+g_{n}\right)}\left(g_{n}\left(x_{n-r_{k}-1} f_{n}-1\right)\right. \\
& \left.\quad+f_{n}\left(\frac{x_{n-r_{k}-1}}{f_{n}}-1\right)\right) .
\end{aligned}
$$

From (40) and (41), we see if we show that $x_{n-r_{k}-1} f_{n}-1$ and $\left(x_{n-r_{k}-1} / f_{n}\right)-1$ have the same sign for $n \in \mathbb{N}$, then $P_{n} Q_{n}$ will be nonpositive.

We consider four cases.

Case 1. $x_{n-r_{k}-1} \geq 1, f_{n} \geq 1$. Clearly in this case $x_{n-r_{k}-1} f_{n}-1 \geq$ 0 . By (H1) and (H2), we have that

$$
\begin{aligned}
1 & \leq f_{n}=\left(f_{n}\right)^{*}=f\left(x_{n-r_{1}-1}^{*}, \ldots, x_{n-r_{k}-1}^{*}\right) \\
& \leq x_{n-r_{k}-1}^{*}=x_{n-r_{k}-1} .
\end{aligned}
$$

Hence $\left(x_{n-r_{k}-1} / f_{n}\right)-1 \geq 0$ and consequently

$$
\left(x_{n-r_{k}-1} f_{n}-1\right)\left(\frac{x_{n-r_{k}-1}}{f_{n}}-1\right) \geq 0 \text {. }
$$

Case 2. $x_{n-r_{k}-1} \geq 1, f_{n} \leq 1$. Since $1 / f_{n} \geq 1$, we obtain $\left(x_{n-r_{k}-1} / f_{n}\right)-1 \geq 0$. On the other hand, by (H1) and (H2), we have

$$
\begin{aligned}
\frac{1}{f_{n}} & =\left(f_{n}\right)^{*}=f\left(x_{n-r_{1}-1}^{*}, \ldots, x_{n-r_{k}-1}^{*}\right) \\
& \leq x_{n-r_{k}-1}^{*}=x_{n-r_{k}-1},
\end{aligned}
$$

so that $x_{n-r_{k}-1} f_{n}-1 \geq 0$. Hence (43) follows in this case.

Case 3. Case $x_{n-r_{k}-1} \leq 1, f_{n} \geq 1$. Then we have that $1 / f_{n} \leq 1$ and consequently $\left(x_{n-r_{k}-1} / f_{n}\right)-1 \leq 0$. On the other hand, we have

$$
f_{n}=\left(f_{n}\right)^{*}=f\left(x_{n-r_{1}-1}^{*}, \ldots, x_{n-r_{k}-1}^{*}\right) \leq x_{n-r_{k}-1}^{*}=\frac{1}{x_{n-r_{k}-1}},
$$

so that $x_{n-r_{k}-1} f_{n}-1 \leq 0$. Hence (43) follows in this case too.

Case 4. Case $x_{n-r_{k}-1} \leq 1, f_{n} \leq 1$. Then $x_{n-r_{k}-1} f_{n}-1 \leq 0$. On the other hand, we have

$$
\frac{1}{f_{n}}=\left(f_{n}\right)^{*}=f\left(x_{n-r_{1}-1}^{*}, \ldots, x_{n-r_{k}-1}^{*}\right) \leq x_{n-r_{k}-1}^{*}=\frac{1}{x_{n-r_{k}-1}},
$$


so that $\left(x_{n-r_{k}-1} / f_{n}\right)-1 \leq 0$. Hence (43) also holds in this case. Thus $P_{n} Q_{n} \leq 0$, for every $n \in \mathbb{N}$.

Assume that $P_{n} Q_{n}=0$, then $P_{n}=0$ or $Q_{n}=0$. Using (40) or (41) along with (43) in any of these two cases, we have that

$$
f_{n}=\frac{1}{x_{n-r_{k}-1}}=x_{n-r_{k}-1}, \quad n \in \mathbb{N} .
$$

Hence $x_{n-r_{k}-1}=1, n \in \mathbb{N}$.

Let

$$
\begin{aligned}
& \widehat{f}_{n}=f\left(y_{n-r_{1}-1}, \ldots, y_{n-r_{k}-1}\right), \\
& \widehat{g}_{n}=g\left(x_{n-m_{1}-1}, \ldots, x_{n-m_{l}-1}\right) .
\end{aligned}
$$

Using the following expressions:

$$
\begin{aligned}
\widehat{P}_{n} & :=\frac{\widehat{f}_{n} \widehat{g}_{n}+1}{\widehat{f}_{n}+\widehat{g}_{n}}-y_{n-r_{k}-1} \\
& =\frac{1}{\widehat{f}_{n}+\widehat{g}_{n}}\left(\widehat{f}_{n} \widehat{g}_{n}\left(1-\frac{y_{n-r_{k}-1}}{\widehat{f}_{n}}\right)+1-y_{n-r_{k}-1} \widehat{f}_{n}\right), \\
\widehat{Q}_{n} & :=\frac{\widehat{f}_{n} \widehat{g}_{n}+1}{\widehat{f}_{n}+\widehat{g}_{n}}-\frac{1}{y_{n-r_{k}-1}} \\
& =\frac{1}{y_{n-r_{k}-1}\left(\widehat{f}_{n}+\widehat{g}_{n}\right)}\left(\widehat{g}_{n}\left(y_{n-r_{k}-1} \widehat{f}_{n}-1\right)\right. \\
& \left.+\widehat{f}_{n}\left(\frac{y_{n-r_{k}-1}}{\widehat{f}_{n}}-1\right)\right),
\end{aligned}
$$

it can be proved similarly that $\widehat{P}_{n} \widehat{Q}_{n} \leq 0$, for every $n \in \mathbb{N}$, and that $\widehat{P}_{n} \widehat{Q}_{n}=0$, if and only if $y_{n-r_{k}-1}=1, n \in \mathbb{N}$.

Finally, let $\left(x^{*}, y^{*}\right)$ be a solution of the system

$$
x^{*}=\frac{f\left(\vec{x}_{k}^{*}\right) g\left(\vec{y}_{l}^{*}\right)+1}{f\left(\vec{x}_{k}^{*}\right)+g\left(\vec{y}_{l}^{*}\right)}, \quad y^{*}=\frac{f\left(\vec{y}_{k}^{*}\right) g\left(\vec{x}_{l}^{*}\right)+1}{f\left(\vec{y}_{k}^{*}\right)+g\left(\vec{x}_{l}^{*}\right)} \text {. }
$$

Then we have that

$$
\begin{aligned}
& 0=\frac{f\left(\vec{x}_{k}^{*}\right) g\left(\vec{y}_{l}^{*}\right)+1}{f\left(\vec{x}_{k}^{*}\right)+g\left(\vec{y}_{l}^{*}\right)}-x^{*} \\
&=\frac{1}{f\left(\vec{x}_{k}^{*}\right)+g\left(\vec{y}_{l}^{*}\right)}\left(f\left(\vec{x}_{k}^{*}\right) g\left(\vec{y}_{l}^{*}\right)\left(1-\frac{x^{*}}{f\left(\vec{x}_{k}^{*}\right)}\right)\right. \\
&\left.+1-x^{*} f\left(\vec{x}_{k}^{*}\right)\right),
\end{aligned}
$$

where $\vec{z}_{j}^{*}=\left(z^{*}, \ldots, z^{*}\right)$ denotes the vector consisting of $j$ copies of $z^{*}$. Then similar to the considerations in Cases (i)(iv), it follows that $f\left(\vec{x}_{k}^{*}\right)=x^{*}=1 / x^{*}$, so that $x^{*}=1$, and similarly it is obtained that $y^{*}=1$. Hence $\left(x^{*}, y^{*}\right)=(1,1)$ is a unique positive equilibrium of system (26).

From all above mentioned and by Proposition 2, we get the result.

\section{Acknowledgments}

S. Stević would like to express his sincere thanks to Professors G. Papaschinopoulos and C. J. Schinas for useful conversations and their help during writing this paper. The first author is supported by the Grant P201/10/1032 of the Czech Grant Agency (Prague). The fourth author is supported by the grant FEKT-S-11-2-921 of Faculty of Electrical Engineering and Communication, Brno University of Technology. This paper is also supported by the Serbian Ministry of Science Projects III 41025, III 44006, and OI 171007.

\section{References}

[1] L. Berg and S. Stević, "On the asymptotics of some difference equations," Journal of Difference Equations and Applications, vol. 18, no. 5, pp. 785-797, 2012.

[2] B. D. Iričanin and S. Stević, "Some systems of nonlinear difference equations of higher order with periodic solutions," Dynamics of Continuous, Discrete \& Impulsive Systems A, vol. 13, no. 3-4, pp. 499-507, 2006.

[3] B. Iričanin and S. Stević, "Eventually constant solutions of a rational difference equation," Applied Mathematics and Computation, vol. 215, no. 2, pp. 854-856, 2009.

[4] N. Kruse and T. Nesemann, "Global asymptotic stability in some discrete dynamical systems," Journal of Mathematical Analysis and Applications, vol. 235, no. 1, pp. 151-158, 1999.

[5] W. Liu, X. Yang, S. Stević, and B. Iričanin, "Part metric and its applications to cyclic discrete dynamical systems," Abstract and Applied Analysis, vol. 2011, Article ID 534974, 16 pages, 2011.

[6] G. Papaschinopoulos and C. J. Schinas, "On a system of two nonlinear difference equations," Journal of Mathematical Analysis and Applications, vol. 219, no. 2, pp. 415-426, 1998.

[7] G. Papaschinopoulos and C. J. Schinas, "On the behavior of the solutions of a system of two nonlinear difference equations," Communications on Applied Nonlinear Analysis, vol. 5, no. 2, pp. 47-59, 1998.

[8] G. Papaschinopoulos and C. J. Schinas, "Invariants for systems of two nonlinear difference equations," Differential Equations and Dynamical Systems, vol. 7, no. 2, pp. 181-196, 1999.

[9] G. Papaschinopoulos and C. J. Schinas, "Invariants and oscillation for systems of two nonlinear difference equations," Nonlinear Analysis: Theory, Methods \& Applications A, vol. 46, no. 7, pp. 967-978, 2001.

[10] G. Papaschinopoulos and C. J. Schinas, "Oscillation and asymptotic stability of two systems of difference equations of rational form," Journal of Difference Equations and Applications, vol. 7, no. 4, pp. 601-617, 2001.

[11] G. Papaschinopoulos and C. J. Schinas, "Global asymptotic stability and oscillation of a family of difference equations," Journal of Mathematical Analysis and Applications, vol. 294, no. 2, pp. 614-620, 2004.

[12] G. Stefanidou, G. Papaschinopoulos, and C. J. Schinas, "On a system of two exponential type difference equations," Communications on Applied Nonlinear Analysis, vol. 17, no. 2, pp. 1-13, 2010.

[13] S. Stević, "Global stability and asymptotics of some classes of rational difference equations," Journal of Mathematical Analysis and Applications, vol. 316, no. 1, pp. 60-68, 2006. 
[14] S. Stević, "On positive solutions of a $(k+1)$-th order difference equation," Applied Mathematics Letters, vol. 19, no. 5, pp. 427431, 2006.

[15] S. Stević, "Existence of nontrivial solutions of a rational difference equation," Applied Mathematics Letters, vol. 20, no. 1, pp. 28-31, 2007.

[16] S. Stević, "Nontrivial solutions of a higher-order rational difference equation," Mathematical Notes, vol. 84, no. 5-6, pp. 718724, 2008.

[17] S. Stević, "On a system of difference equations," Applied Mathematics and Computation, vol. 218, no. 7, pp. 3372-3378, 2011.

[18] S. Stević, "On a solvable rational system of difference equations," Applied Mathematics and Computation, vol. 219, no. 6, pp. 28962908, 2012.

[19] S. Stević, "On a third-order system of difference equations," Applied Mathematics and Computation, vol. 218, no. 14, pp. 7649-7654, 2012.

[20] S. Stević, "On some solvable systems of difference equations," Applied Mathematics and Computation, vol. 218, no. 9, pp. 50105018, 2012.

[21] A. C. Thompson, "On certain contraction mappings in a partially ordered vector space," Proceedings of the American Mathematical Society, vol. 14, pp. 438-443, 1963.

[22] X. Yang, M. Yang, and H. Liu, "A part-metric-related inequality chain and application to the stability analysis of difference equation," Journal of Inequalities and Applications, vol. 2007, Article ID 19618, 9 pages, 2007.

[23] I. Yalcinkaya, "On the global asymptotic stability of a secondorder system of difference equations," Discrete Dynamics in Nature and Society, vol. 2008, Article ID 860152, 12 pages, 2008.

[24] N. Yalcinkaya and C. Çinar, "Global asymptotic stability of a system of two nonlinear difference equations," Fasciculi Mathematici, no. 43, pp. 171-180, 2010.

[25] I. Yalcinkaya, C. Cinar, and D. Simsek, "Global asymptotic stability of a system of difference equations," Applicable Analysis, vol. 87, no. 6, pp. 677-687, 2008. 


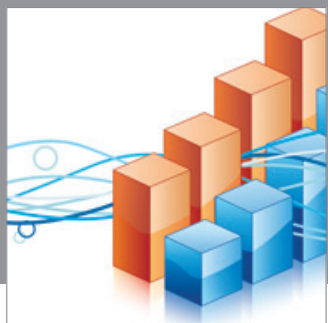

Advances in

Operations Research

mansans

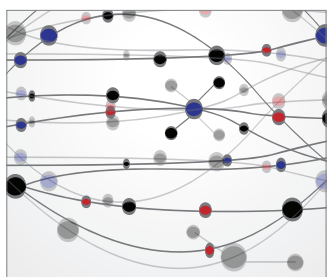

The Scientific World Journal
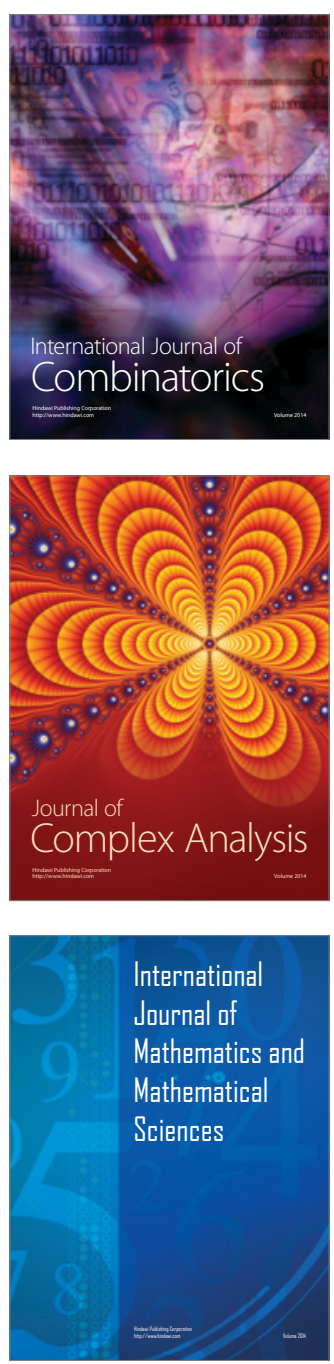
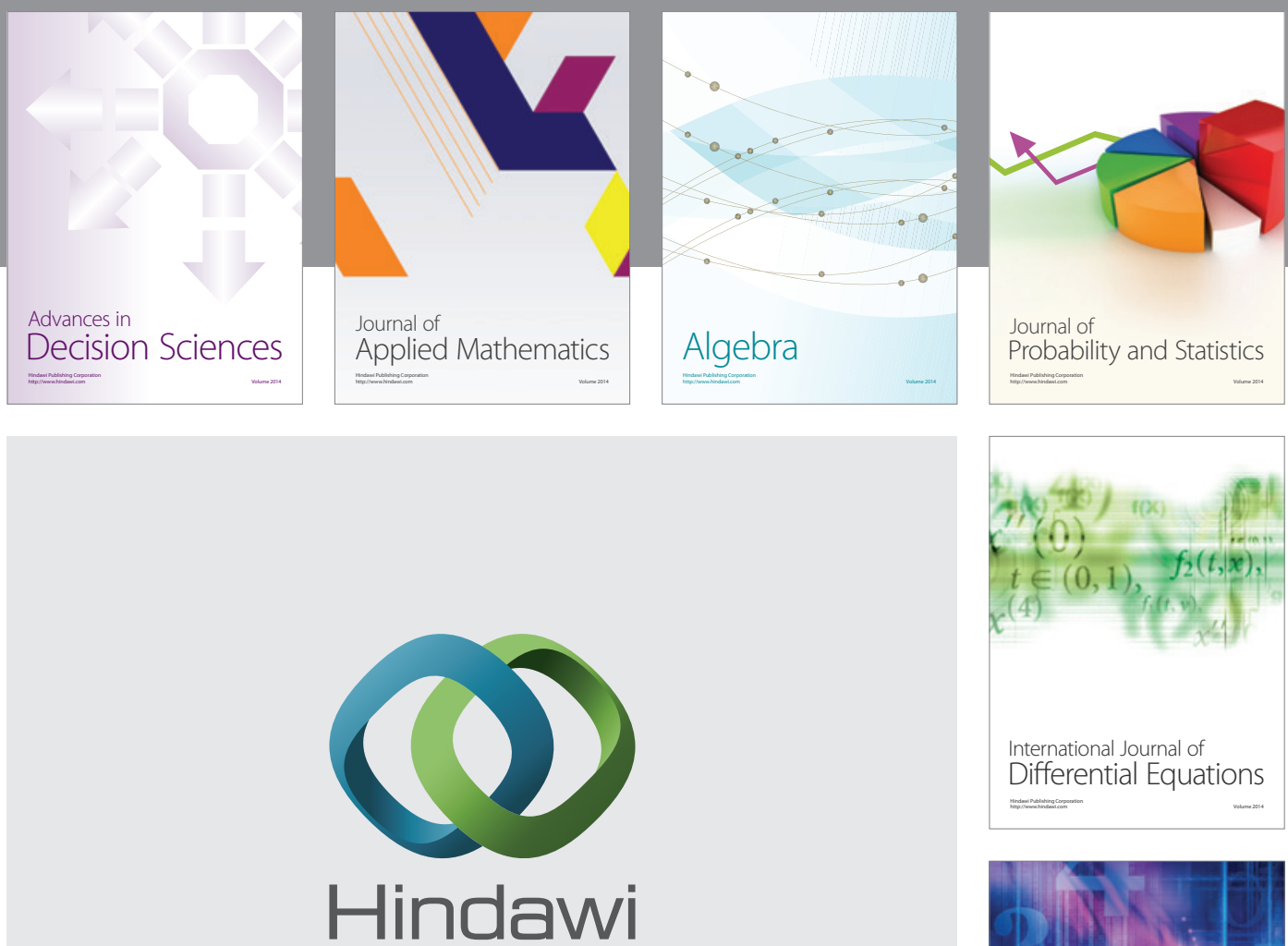

Submit your manuscripts at http://www.hindawi.com
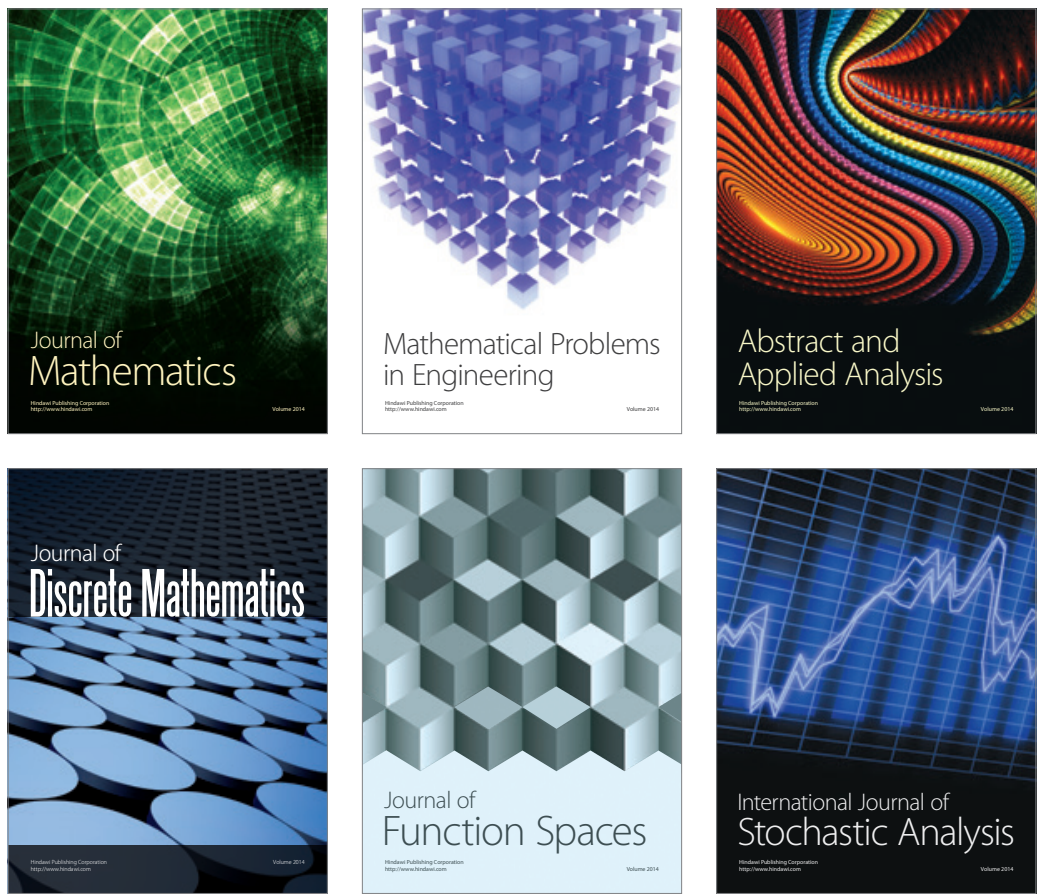

Journal of

Function Spaces

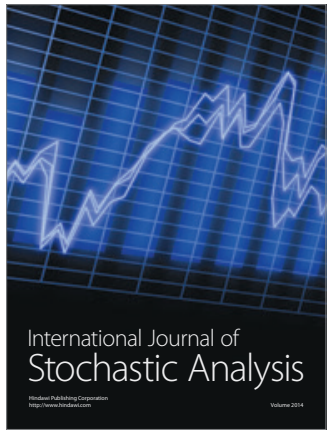

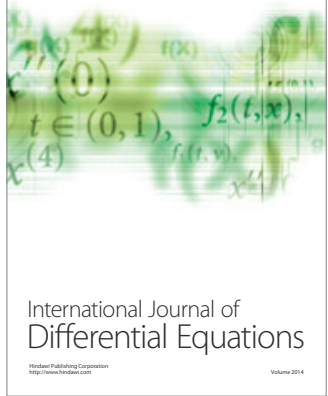
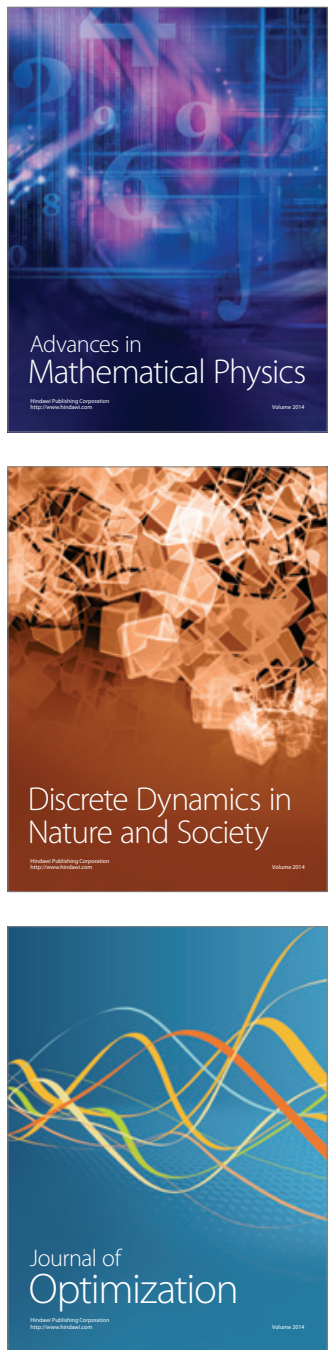